\title{
Sensitivity Bond Graphs
}

\author{
Peter J Gawthrop ${ }^{a}$ \\ ${ }^{a}$ Centre for Systems and Control and Department of Mechanical Engineering, \\ University of Glasgow, GLASGOW. G12 8QQ Scotland. \\ P.Gawthrop@eng.gla.ac.uk
}

\begin{abstract}
A sensitivity bond graph, of the same structure as the system bond graph, is shown to provide a simple and effective method of generating sensitivity functions of use in optimisation. The approach is illustrated in the context of partially-known system parameter and state estimation.
\end{abstract}

Key words: Bond graphs; sensitivity; optimisation; parameter estimation; partially-knowm systems 


\section{Introduction}

Optimisation [1] has always been a fundamental technique in control and parameter estimation of dynamic systems. Such methods rely on a model of the corresponding physical system. In some application areas (for example process control) physical, or first principles, models are hard to come by and so empirical models tend to be used. However in other application areas, in particular mechatronics, physical models are more readily available. There are a number of techniques for building control-relevant models but the bond graph $[2,3,4,5]$ approach is well established and has been suggested as a basis for control design $[6,7,8]$.

However, optimisation is eased by having a sensitivity model in addition to the model itself [1]. This paper develops the idea of sensitivity bond graphs $[9,10]$ to provide a basis for model-based optimisation for the identification of systems for which a bond graph model is available. Sensitivity theory of dynamic systems and its application is well established and summarised in the textbooks $[11,12]$. There are many applications of sensitivity methods to systems and control problems including: system optimisation [9], controller tuning $[13,14,15]$ and parameter estimation [16]. The particular class of dynamic systems described by electrical networks has its own techniques [17] based on the adjoint circuit approach.

A basic theoretical result of sensitivity theory $[11,12]$ is that, given the timeinvariant ordinary differential equation with state $X \in \Re^{n}$, initial state $X_{0}$, input $u$ and constant parameter vector $\theta \in \Re^{n_{\theta}}$ of the form:

$$
\begin{cases}\dot{x} & =f(x, u, \theta) \\ y & =g(x, \theta) \\ x(0) & =x_{0}\end{cases}
$$

then the state sensitivity functions $x^{j}=\frac{\partial^{j} x}{\partial \theta_{j}}$ and the output sensitivity functions $y_{i}^{j}=\frac{\partial^{j} y}{\partial \theta_{j}}$ are the solutions of the $n_{\theta}$ linear time-varying ordinary differential equations:

$$
\begin{cases}\dot{x^{j}} & =A(x, u) x^{j}+U(x, u) \\ y^{j} & =C(x, u) x^{j} \\ x^{j}(0) & =x_{0}^{j}\end{cases}
$$

Except in special cases [18], it is necessary to use $n_{\theta}$ systems of the form of Equation 2, together with original system of Equation 1, to compute the $n_{\theta}$ sensitivity functions.

In contrast to standard sensitivity theory $[11,12]$, which operates at the system ordinary differential equation level, this paper (in common with $[9,10]$ ) devel- 
ops a component-based approach which, given the sensitivity models of each component, builds the system sensitivity ordinary differential equation from that of the system components. Linearisation theory is a special case of sensitivity theory. In the context of linearisation, Karnopp [19] has stated: "Rather than treating linearized systems as abstract sets of equations, we here look for structural analogies between non-linear components relating total system variables and linearized models relating incremental system variables"; with "linearized" replaced by "sensitivity" this statement summarises the aim of this paper. In particular, we follow Karnopp [19] in using a bond graph $[2,3,4,5]$ approach to sensitivity theory.

Cabanellas, Felez and Vera [9] have established a bond graph interpretation of sensitivity and applied it to system optimisation. This approach has the advantage of having a clear physical interpretation and focuses on the class of parameters associated with the constitutive relationships of bond graph components. The same ideas have also been presented (apparently independently) by Roe and Thoma [10].

Cabanellas, Felez and Vera [9] also demonstrate the advantages of algebraic generation of sensitivity functions as opposed to numerical approximations. Indeed, symbolic methods for nonlinear systems modelling, analysis and optimisation are currently strong research areas [20] driven by the ready availability of symbolic computational tools. In particular, the bond graph approach $[2,5]$ has been used to generate models applicable to control design [8].

In this paper the work of Cabanellas, Felez and Vera [9] is extended. In particular, it is shown that by making use of vector bonds and vector junctions, the sensitivity bond graph is structurally identical to the system bond graph. This is useful because the bond graph of the sensitivity system can be derived directly (and hence automatically) from the system bond graph. This sensitivity bond graph can be used not only as a way to derive the system sensitivity equations, but also as a means of directly analysing the system sensitivity properties on the bond graph itself.

To illustrate the application of the sensitivity bond graph theory of this paper to a concrete optimisation example, the identification of partially-known systems is considered. Many engineering systems of interest to the control engineer are partially known in the sense that the system structure, together with some system parameters are known, but some system parameters are unknown. This gives rise to a problem of parameter estimation when values for the unknown parameters are to be determined from experimental data comprising measurements of system inputs and outputs. There is a considerable literature in the area including $[21,22,23,24,25]$. Although in special cases such identification may be linear-in-the parameters [21] or polynomial-in-the parameters $[24,25]$ in general the problem is nonlinear-in-the parameters and 
gives rise to a nonlinear least-squares problem [1]. This means that, in general, the resultant optimisation problem is not quadratic or polynomial, and may even be non-convex; such nonlinear least-squares optimisation problems are conveniently solved using Gauss-Newton or Levenberg-Marquardt algorithms [1]. In such cases, the optimisation task is eased by knowing a symbolic expression for (rather than deducing numerically) the derivative of the error function with respect to the unknown system parameters. It therefore follows that partially known system identification is one application of optimisation using sensitivity bond graphs. This is used as an example application in this paper.

The outline of the paper follows. Section 2 discusses the sensitivity of systems described by bond graphs and shows that a new type of bond graph, the sensitivity bond graph, represents the system sensitivity whilst having the same structure and causality as the system bond graph. Section 3 reviews the Gauss-Newton optimisation technique [1] and shows how the sensitivity bond graph can be used to generate the corresponding gradient information. Section 4 illustrates the approach via combined state and parameter estimation of a nonlinear system. Section 5 makes some concluding remarks.

\section{Sensitivity Bond Graphs}

A bond graph component is associated with a constitutive relationship (or CR) which relates the time-varying signals within the component and the timeinvariant parameters associated with the system within which the component lies. Thus the CR for the $i$ th component (associated with $n_{v_{i}}$ signals and $n_{\theta}$ parameters) of a system can be written as:

$$
\Phi_{i}\left(v_{i}(t), \theta\right)=0
$$

where $v_{i} \in \Re^{n_{v_{i}}}$ contains the component signals and $\theta \in \Re^{n_{\theta}}$ contains the system parameters.

In the special case that the $\mathrm{CR}$ is linear; it can be written as:

$$
A_{i}(\theta)^{T} v_{i}(t)=0
$$

where the time-invariant vector $A_{i}(\theta) \in \Re^{n_{v_{i}}}$.

In this paper, the sensitivity ${ }^{j} v_{i} \in \Re^{n_{v_{i}}}$ of the signal vector $v_{i}$ with respect to the $j$ th component $\theta_{j}$ of the parameter vector $\theta$ is of interest. In particular, ${ }^{j} v_{i}$ is defined as:

$$
{ }^{j} v_{i}=\frac{\partial v_{i}}{\partial \theta_{j}}
$$


It follows from Equation 3 that $\frac{d \Phi_{i}\left(v_{i}(t), \theta\right)}{d \theta}=0$ and so the sensitivity CR ${ }^{j} \phi_{i}\left({ }^{j} v_{i}(t), v_{i}(t), \theta\right)$ becomes:

$$
\begin{aligned}
{ }^{j} \phi_{i}\left({ }^{j} v_{i}(t), v_{i}(t), \theta\right) & =\frac{\partial \Phi_{i}\left(v_{i}(t), \theta\right)^{T}}{\partial v_{i}}{ }^{j} v_{i}(t) \\
& +\frac{\partial \Phi_{i}\left(v_{i}(t), \theta\right)}{\partial \theta_{j}}=0
\end{aligned}
$$

Equation 6 is the sensitivity $\mathrm{CR}$ with respect to the $j$ th parameter. It has the following important properties:

(1) The first term of the right-hand side of Equation 6 represents the linearised (about $\left.v_{i}(t)\right)$ CR relating the sensitivity functions ${ }^{j} v_{i}(t)$. In other words, it is a linear CR modulated by the variables $v_{i}(t)$ associated with the system itself. It can be written as the summation:

$$
\frac{\partial \Phi_{i}\left(v_{i}(t), \theta\right)^{T}}{\partial v_{i}}{ }^{j} v_{i}(t)=\sum_{k=1}^{n_{v_{i}}} \frac{\partial \Phi_{i}\left(v_{i}(t), \theta\right)^{T}}{\partial v_{i k}}{ }^{j} v_{i k}(t)
$$

where $k$ indexes the $n_{v_{i}}$ elements of $v_{i}$ and ${ }^{j} v_{i}$.

(2) The second term of the right-hand side of Equation 6 represents an additional input to the sensitivity $\mathrm{CR}$ dependent on the variables $v_{i}(t)$ associated with the system itself.

(3) The sensitivity $\mathrm{CR}$ of Equation 6 is local to the the component in the sense that the only variables appearing in Equation 6 are $v_{i}(t)$ and ${ }^{j} v_{i}(t)$.

(4) The $j$ th sensitivity CR of Equation 6 does not depend on ${ }^{l} v(t)$ for $l \neq j$.

(5) If the $i$ th $\mathrm{CR} \Phi_{i}\left(v_{i}(t), \theta\right)$ does not depend on $\theta_{j}$ then the second term of Equation 6 is zero and there is no explicit coupling between the actual and sensitivity systems (though there will be implicitly if $\Phi_{i}\left(v_{i}(t), \theta\right)$ is nonlinear.

(6) If $\Phi_{i}\left(v_{i}(t), \theta\right)$ is linear in $v_{i}$ (Equation 4, then Equation 6 becomes:

$$
A_{i}^{T}(\theta){ }^{j} v_{i}(t)+\frac{\partial A_{i}^{T}(\theta)}{\partial \theta_{j}} v_{i}(t)=0
$$

(7) If the conditions of both items 5 and 6 hold; then the CRs of the actual and sensitivity components are identical and uncoupled.

For these reasons, it is possible to encapsulate two CRs: the system CR of Equation 3 and the $j$ th sensitivity CR of Equation 6 within in a single component containing $2 n_{v_{i}}$ variables: those contained in $v_{i}$ and ${ }^{j} v_{i}$. If the original component had $N$ ports, the new sensitivity component (s-component) therefore has $2 N$ ports. More conveniently, each port on the original component is replaced by a sensitivity port (or s-port) which carries not only the effort/flow pair $e$ and $f$ but also the corresponding sensitivity pair ${ }^{j} e$ and ${ }^{j} f$. Such ports 
may be considered to be connected by a sensitivity bond (s-bond) which encapsulates the energy bond carrying $e$ and $f$ with the pseudo-bond carrying ${ }^{j} e$ and ${ }^{j} f$.

The encapsulated CR (Equations 3 and 6) refer to only the $j$ th of $n_{\theta}$ parameters. To obtain the bond graph for all $n_{\theta}$ parameters there are two possible approaches:

(1) Derive the $n_{\theta}$ CRs of Equation 6 and encapsulate $n_{\theta}$ CRs together with the component $\mathrm{CR}$ in each component and extend each port and s-bond to correspond to $n_{\theta}+1$ bonds.

(2) Define the scalar pseudo effort and flow variables ${ }^{\lambda} e$ and ${ }^{\lambda} f$ as

$$
\begin{aligned}
& { }^{\lambda} e=\sum_{j=1}^{n_{\theta}} \Lambda_{j}{ }^{j} e \\
& { }^{\lambda} f=\sum_{j=1}^{n_{\theta}} \Lambda_{j}{ }^{j} f
\end{aligned}
$$

where $\Lambda \in \Re^{n_{v_{i}}}$ is a vector of weighting coefficients. In this case, define:

$$
\phi_{i}\left({ }^{\lambda} v_{i}(t), v_{i}(t), \theta\right)=\sum_{j=1}^{n_{\theta}} \lambda_{j}^{j} \phi_{i}\left({ }^{j} v_{i}(t), v_{i}(t), \theta\right)
$$

where $\lambda_{j}$ is the $j$ th component of $\Lambda$.

The latter approach has the advantage of giving a simpler bond graph where all s-bonds encapsulate two bonds: the energy bond carrying $e$ and $f$ with the pseudo-bond carrying ${ }^{\lambda} e$ and ${ }^{\lambda} f$. It is parameterised by $\Lambda$ and any particular sensitivity function can be extracted by setting $\Lambda=\Lambda_{j}$, the unit vector with the $j$ th element unity and the rest zero. This is the approach used in the remainder of this paper.

Having created the sensitivity bond graph using this approach, the resultant dynamic system will $n_{y}$ outputs corresponding to the underlying bond graph together with a further $n_{y}$ outputs corresponding to the sensitivity outputs denoted by ${ }^{\lambda} y \in \Re^{n_{y}}$.

The output sensitivity function $y_{\theta}$ can be constructed by solving the ordinary differential equation corresponding to the sensitivity system $n_{\theta}$ times with $\Lambda=\Lambda_{j}$ for $0 \leq j \leq n_{\theta}$. 


\subsection{Some sensitivity components}

It follows from the discussion of Section 2 that any Bond Graph component has an s-component equivalent. This section provides some examples, including those which will be used in the sequel.

\subsubsection{A linear one-port $\boldsymbol{R}$ component}

The standard linear bond graph $\mathbf{R}$ component has a single port with effort $e_{i}$ and flow $f_{i}$ covariables related by the $\mathrm{CR}$

$$
\Phi_{i}\left(v_{i}(t), \theta\right)=A_{i}(\theta)^{T} v_{i}(t)=e-r f=0
$$

That is, in the notation of Equation 4

$$
A_{i}(\theta)=\left(\begin{array}{c}
1 \\
-r
\end{array}\right) ; v_{i}=\left(\begin{array}{c}
e_{i} \\
f_{i}
\end{array}\right)
$$

$\frac{\partial A_{i}^{T}(\theta)}{\partial r}=(0-1)^{T}$ and so the sensitivity CR of Equation 7 can be written as:

$$
\begin{cases}\frac{\partial e_{i}}{\partial r}-r \frac{\partial f_{i}}{\partial r}-f_{i}=0 & \text { if } \theta_{j} \equiv r \\ \frac{\partial e_{i}}{\partial r}-r \frac{\partial f_{i}}{\partial r}=0 & \text { if } \theta_{j} \not \equiv r\end{cases}
$$

or alternatively:

$$
{ }^{\lambda} e_{i}-r{ }^{\lambda} f_{i}-\lambda_{j} f_{i}=0
$$

The corresponding sensitivity component is a linear two port $\mathbf{R}$ with $\mathrm{CR}$ given by Equations 10 and 13. The upper part of Figure 1 shows this two port $\mathbf{R}$; the lower part of Figure 1 shows the encapsulated version.

\subsubsection{A nonlinear one-port $\boldsymbol{R}$ component}

Consider a nonlinear $\mathbf{R}$ component with a single port with effort $e$ and flow $f$ covariables related by the $\mathrm{CR}$

$$
\Phi_{i}\left(v_{i}(t), \theta\right)=e_{i}-\beta f_{i}^{\alpha}=0
$$

This corresponds to a typical hydraulic resistance with unidirectional flow $\left(f_{i}>0\right)$. 
Using $v_{i}$ as defined in 11, it follows that

$$
\frac{\partial \Phi_{i}\left(v_{i}(t), \theta\right)}{\partial v_{i}}=\left(\begin{array}{c}
1 \\
-\beta \alpha f_{i}^{\alpha-1}
\end{array}\right)
$$

Defining

$$
\theta=\left(\begin{array}{l}
\alpha \\
\beta
\end{array}\right)
$$

It follows that

$$
\frac{\partial \Phi_{i}\left(v_{i}(t), \theta\right)}{\partial \theta}=\left(\begin{array}{c}
-\beta \ln f \\
-1
\end{array}\right) f_{i}^{\alpha}
$$

Hence

$$
\phi_{i}\left({ }^{\lambda} v_{i}(t), v_{i}(t), \theta\right)={ }^{\lambda} e_{i}-\alpha f_{i}^{\alpha-1}{ }^{\lambda} f_{i}-\lambda_{1} \beta f_{i}^{\alpha} \ln f_{i}-\lambda_{2} f_{i}^{\alpha}=0
$$

The CR represented by Equations 14 and 18 is convenient for computing $e_{i}$ and ${ }^{\lambda} e_{i}$ in terms of $f_{i}$ and ${ }^{\lambda} f_{i}$, but only has an implicit solution for $f_{i}$ and ${ }^{\lambda} f_{i}$ in terms of $e_{i}$ and ${ }^{\lambda} e_{i}$. However an explicit expression for $f_{i}$ and ${ }^{\lambda} f_{i}$ may be obtained by rewriting Equation 14 to give the alternative $\operatorname{CR}\left(\Phi_{i}^{a}\left(v_{i}(t), \theta\right)\right)$

$$
\Phi_{i}^{a}\left(v_{i}(t), \theta\right)=\left(\frac{e_{i}}{\beta}\right)^{\frac{1}{\alpha}}-f_{i}=0
$$

Equation 18 may then be replaced by the alternative sensitivity $\mathrm{CR}\left(\phi_{i}^{a}\left({ }^{\lambda} v_{i}(t), v_{i}(t), \theta\right)\right)$

$$
\begin{aligned}
\phi_{i}^{a}\left({ }^{\lambda} v_{i}(t), v_{i}(t), \theta\right) & ={ }^{\lambda} f_{i} \\
& -\left(\frac{e_{i}}{\beta}\right)^{\frac{1}{\alpha}}\left[+\frac{1}{\alpha e_{i}}{ }^{\lambda} e_{i}-\lambda_{1} \frac{1}{\alpha^{2}} \ln \left(\frac{e_{i}}{\beta}\right)-\lambda_{2} \frac{1}{\alpha \beta}\right] \\
& =0
\end{aligned}
$$

The corresponding sensitivity component is a nonlinear two port $\mathbf{R}$ with $\mathrm{CR}$ given by Equations 14 and 18 or by Equations 19 and 20. The upper part of Figure 1 shows this two port $\mathbf{R}$; the lower part of Figure 1 shows the encapsulated version.

\subsubsection{A linear one-port $\boldsymbol{C}$ component}

The standard linear bond graph $\mathbf{R}$ component has a single port with effort $e_{i}$ and integrated flow $q_{i}$ covariables related by the CR

$$
\Phi_{i}\left(v_{i}(t), \theta\right)=A_{i}(\theta)^{T} v_{i}(t)=e_{i}-a\left(q_{i}+q_{i 0}\right)=0
$$


where $a$ is a constant parameter and $q_{i 0}$ is the initial state.

If the parameter vector $\theta$ is taken as

$$
\theta=\left(\begin{array}{c}
a \\
q_{i 0}
\end{array}\right)
$$

then the sensitivity $\mathrm{CR}$ of Equation 7 can be written as:

$$
\phi_{i}\left({ }^{\lambda} v_{i}(t), v_{i}(t), \theta\right)={ }^{\lambda} e_{i}-a{ }^{\lambda} q_{i}-\lambda_{1}\left(q_{i}+q_{i 0}\right)-\lambda_{2} a
$$

The corresponding sensitivity component is a linear two port $\mathbf{C}$ with $\mathbf{C R}$ given by Equations 20 and 22 .

\subsubsection{A nonlinear one-port $\boldsymbol{C}$ component}

An open right-conical tank containing of incompressible liquid can be regarded as a nonlinear $\mathbf{C}$ component with $\mathbf{C R}$ :

$$
\Phi_{i}\left(v_{i}(t), \theta\right)=e_{i}-\rho g\left(\frac{12}{\pi}\left(q_{i}+q_{i 0}\right)\right)^{\frac{1}{3}}=0
$$

where $q$ is the integrated flow variable, $\rho$ is the fluid density, $g$ the gravitational constant and $q_{i 0}$ the initial volume of liquid. If the parameter vector $\theta$ is taken as

$$
\theta=\left(\begin{array}{c}
\rho \\
q_{i 0}
\end{array}\right)
$$

then the sensitivity $\mathrm{CR}$ of Equation 7 can be written as:

$$
\begin{aligned}
\phi_{i}\left({ }^{\lambda} v_{i}(t), v_{i}(t), \theta\right) & ={ }^{\lambda} e_{i} \\
& -\left(\frac{12}{\pi}\left(q_{i}+q_{i 0}\right)\right)^{\frac{1}{3}}\left[\left({ }^{\lambda} q_{i}+\lambda_{2}\right) \rho g \frac{1}{3\left(q_{i}+q_{i 0}\right)}+\lambda_{1} g\right]
\end{aligned}
$$

The corresponding sensitivity component is a nonlinear two port $\mathbf{C}$ with $\mathrm{CR}$ given by Equations 23 and 25. The upper part of Figure 1 (with $\mathbf{R}$ replaced by $\mathbf{C}$ ) shows this two port $\mathbf{C}$; the lower part of Figure 1 (with $\mathbf{R}$ replaced by C) shows the encapsulated version. 


\section{Optimisation}

As discussed by Cabanellas, Felez and Vera [9], one application of sensitivity methods is optimisation. This paper considers optimisation problems of the form:

$$
\min _{\theta} J(\theta)
$$

where $\theta \in \Re^{n_{\theta}}$ is the parameter vector and the cost function $J(\theta)$ is

$$
J(\theta)=\frac{1}{2} \int_{0}^{T} e^{T}(t) Q(t) e(t) d t
$$

where $Q(t) \in \Re^{n_{y} \times n_{y}}$ is a positive semi-definite weighting function and the error $e(t) \in \Re^{n_{y}}$ is

$$
e(t)=[y(t, \theta)-z(t)]
$$

$y(t) \in \Re^{n_{y}}$ the system output and $z(t) \in \Re^{n_{y}}$ a function of time. In general, $y(t)$ is not linear in $\theta$ and so $J(\theta)$ is not in general quadratic in $\theta$.

In the context of real-time control and estimation and so optimisation speed is of the essence. Therefore, rapid convergence combined with simplicity is desirable. As, using the sensitivity bond graph approach, gradient information is cheaply available, this suggests the use of methods which make use of gradient information. For these reasons, out of the plethora of methods available (see for example $[1,26])$, we choose the Gauss-Newton method. Further research may yield alternative choices, but our experience so far has been good.

Differentiation of Equations 25 and 27 with respect to $\theta$ relate the gradient $J_{\theta}$ of $J$ (with respect to $\theta$ ) to the corresponding gradient $y_{\theta}(t)$ and the output $y(t)$ as:

$$
J_{\theta}=\int_{0}^{T}[y(t, \theta)-z(t)]^{T} Q(t) y_{\theta}(t) d t
$$

The Gauss-Newton approach approximates the second derivative $J_{\theta \theta}$ of the cost function by:

$$
J_{\theta \theta} \approx \hat{J}_{\theta \theta}=\int_{0}^{T} y_{\theta}(t) Q(t) y_{\theta}^{T}(t) d t
$$

Thus both $J_{\theta}$ and $\hat{J}_{\theta \theta}$ can be derived from the outputs $y(t)$ and $y_{\theta}(t)$ of the sensitivity bond graph system described in Section 2.

The optimisation algorithm is then to repeatedly compute:

$$
\theta:=\theta-\Delta \theta
$$

until some convergence criterion is satisfied where $\Delta \theta$ is the solution of the set of linear equations:

$$
\hat{J}_{\theta \theta} \Delta \theta=J_{\theta}
$$


Two simple modifications of this method give additional robustness in the face of difficult optimisation problems $[1,26]$ :

(1) Equation 31 is solved via a singular value decomposition based pseudo inverse and

(2) A check is made that the cost function decreases at each step; if it doesn't, the step length is halved (whilst retaining the step direction) until it does.

This approach has proved effective for this example. However related nonlinear least-squares algorithms (such as the Levenberg-Martquardt algorithm [1]) may be equally or more effective.

\section{Example}

This example has appeared previously [27] but is extended here to include state estimation and is solved using the novel methods of this paper rather than the preliminary approach given in reference [27].

Figure 2 shows two identical right-conical tanks of liquid and two identical pipes; Figure 3 gives the corresponding bond graph. It is assumed that the pipes can be modelled using the CRs of Section 2.1.2 (Equations 19 and 20) (and therefore that the flow is unidirectional) and the tanks by the CRs of Section 2.1.4 (Equations 23 and 25). The system input $u(t)$ is the inflow, and the system output $y(t)$ the pressure at the base of the right-hand tank.

Figure 3 gives the sensitivity bond graph of the system. The $\mathbf{s R}$ and $\mathbf{s C}$ components are as discussed in Section 2.1.2 and 2.1.4 respectively. The $\mathbf{S f}$ component imposes a flow $u(t)$ on the flow component of the vector bond and zero (corresponding to the fact that $u(t)$ is independent of $\theta$ ) on the sensitivity flow variable. The sDe component measures the output effort $y(t)$ together with the corresponding sensitivity signal.

As discussed in Section 2, the bonds (indicated by the bold lines) are vector bonds carrying sensitivity efforts ${ }^{\lambda} e$ and flows ${ }^{\lambda} f$ in addition to efforts $e$ and flows $f$.

For the purposes of this example, it was assumed that $\rho g=1$ but that the initial volumes of liquid in each tank $q_{10}=V_{1}$ and $q_{20}=V_{2}$ are unknown. It is further assumed that the pipe parameters $r$ and $\alpha$ are unknown. Thus the 
parameter vector $\theta$ is chosen as:

$$
\theta=\left(\begin{array}{c}
V_{1} \\
V_{2} \\
\alpha \\
\beta
\end{array}\right)
$$

The system equations were deduced from the bond graph of Figure 3 using the MTT software [28] using symbolic algebra methods [29] to deduce the sensitivity CRs of Equations 20 and 25. These equations, together with Euler integration code were automatically translated into $\mathrm{C}$ and compiled.

The system was simulated using Euler integration with a step size of 0.01 up to time $T=10$ and using parameters:

$$
\theta=\left(\begin{array}{l}
V_{1} \\
V_{2} \\
\alpha \\
\beta
\end{array}\right)=\left(\begin{array}{c}
0.5 \\
0.25 \\
1.5 \\
1.0
\end{array}\right)
$$

The tank inflow was $u=1.0$.

Figure 5 shows the system output as a function of time. This data was used as the actual system output corresponding to $z(t)$ in Equation 27.

The shape of the graph is explained as follows. The initial output (the pressure at the base of the second tank) is given by Equation 23 as:

$$
y(0)=\rho g\left(\frac{12}{\pi} V_{2}\right)^{\frac{1}{3}} \approx 0.98
$$

The final output is governed by the pressure drop across the second pipe at the steady-state flow rate $f_{\infty}=1$ corresponding to the unit input flow. Using Equation 14

$$
y(\infty)=\beta f_{\infty}^{\alpha}=1
$$

$y(t)$ in Figure 5 is asymptotic to this value.

Figure 6 shows the corresponding four sensitivity functions plotted against time. Of the four parameters, the initial output $y(0)$ is dependent only on $V_{2}$, thus the sensitivity with respect to $V_{1}, \alpha$ and $\beta$ is initially zero. From Equation 25

$$
y_{V_{1}}(0)=\left(\frac{12}{\pi} V_{1}\right)^{\frac{1}{3}}\left[\rho g \frac{1}{3 V_{1}}\right] \approx 1.3
$$


Thus three of the sensitivity function start at zero, whilst that with respect to $V_{2}$ starts at about 1.3. In the steady-state, $y(t)$ only depends on the flow $f_{\infty}=1$ though the second pipe, thus the sensitivity with respect to $V_{1}$ and $V_{2}$ is zero. From Equation 18, the sensitivity of the steady-state output $y(t)$ with respect to $\alpha$ is

$$
y_{\alpha}(\infty)=\beta f_{\infty}^{\alpha} \ln f_{\infty}=0
$$

and the sensitivity of the steady-state output $y(t)$ with respect to $\beta$ is

$$
y_{\beta}(\infty)=f_{\infty}^{\alpha}=1
$$

Thus three of the sensitivity functions are asymptotic to zero, and that with respect to $\beta$ is asymptotic to unity.

Using different parameters $\hat{\theta}$, the same code was used to generate the model output $y(t, \hat{\theta})$ together with the four sensitivity functions contained in the vector $y_{\theta}(t, \hat{\theta})$ The optimisation method of Section 3 was used to determine the parameter estimate $\hat{\theta}$. The starting value of $\hat{\theta}$ was

$$
\hat{\theta}=\left(\begin{array}{l}
V_{1} \\
V_{2} \\
\alpha \\
\beta
\end{array}\right)=\left(\begin{array}{l}
2.0 \\
1.0 \\
1.0 \\
2.0
\end{array}\right)
$$

Figure 7 shows the parameter estimates $\hat{\theta}$ plotted against iteration number. The initial estimates correspond to Equation 39, and the estimates converge to those given in Equation 33.

Figure 8 shows the estimated system output plotted against time corresponding to the initial value of $\hat{\theta}$ and the estimates at iterations 1, 5 and 10 together with the system response. That corresponding to iteration 10 is almost identical to the system response.

Figures 7 and 8 demonstrate that the method is effective in this case.

\section{Conclusion}

A sensitivity bond graph, of the same structure as the system bond graph, is shown to provide a simple and effective method of generating sensitivity functions of use in optimisation.

One application of such optimisation in the context of partially-known system 
parameter and state estimation is given and illustrated using a highly nonlinear system. This sensitivity-based optimisation approach is also relevant to model-based predictive control; this aspect has also been investigated [30].

\section{Acknowledgements}

This work was accomplished whilst the author was a visitor at the Centre for Integrated Dynamics and Control, University of Newcastle, New South Wales. He would like to thank Prof. Graham Goodwin for providing an excellent work environment and Dr. Will Heath for useful insights into optimisation methods.

I would also like to thank Prof. David Murray-Smith of Glasgow University for introducing me to sensitivity methods and Prof. Job Van Amerongen of the University of Twente, Dr Tomas McKelvey of the University of Linköping and Dr. Eric Ronco of the University of Sydney for helpful discussions and advice.

\section{References}

[1] R. Fletcher. Practical Methods of Optimization. 2nd Edition. Wiley, Chichester, 1987.

[2] D. C. Karnopp, D. L. Margolis, and R. C. Rosenberg. System Dynamics: A Unified Approach. John Wiley, 1990.

[3] J. U. Thoma. Simulation by bond graphs. Springer-Verlag, Berlin, 1990.

[4] F. E. Cellier. Continuous system modelling. Springer-Verlag, 1991.

[5] P. J. Gawthrop and L. P. S. Smith. Metamodelling: Bond Graphs and Dynamic Systems. Prentice Hall, Hemel Hempstead, Herts, England., 1996.

[6] D. C. Karnopp. Bond graphs in control: Physical state variables and observers. J. Franklin Institute, 308(3):221-234, 1979.

[7] D. C. Karnopp. Actively controlled systems: - an ideal application area for bond graph modelling (plenary address). In Cellier and Granda [31], page 3.

[8] P. J. Gawthrop. Physical model-based control: A bond graph approach. Journal of the Franklin Institute, 332B(3), 285-305 1995.

[9] J. M. Cabanellas, J. Felez, and C. Vera. A formulation of the sensitivity analysis for dynamic systems optimisation based on pseudo bond graphs. In Cellier and Granda [31], pages 135-144. 
[10] P.H. Roe and J.U. Thoma. A new Bond Graph approach to sensitivity analysis. In I Troch and F. Breitenecker, editors, Proceedings of the 3rd IMACS Symposium on Mathematical Modelling, pages 743-746, Vienna, Austria, February 2000. ARGESIM.

[11] R. Tomović and M. Vukobratović. General Sensitvity Theory. Number 35 in Modern analytic and computational methods in science and mathematics. Elsevier, New York, 1972.

[12] Paul M. Frank. Introduction to System Sensitivity Theory. Academic Press, New York, 1978.

[13] J. Van Amerongen and A. J. Udink ten Cate. Model reference autopilots for ships. Automatica, 11:441-449, 1975.

[14] D.J. Winning, E.H.T. El-Shirbeeny, E.C. Thompson, and D.J. Murray-Smith. Sensitivity method for online optimisation of a synchronous generator excitation controller. Proceedings IEE Part D: Control Theory and Applications, 124(7):631-638, July 1977.

[15] I. Oppen, Mingrui Gong, and D. J. Murray-Smith. The development of tuning techniques for single-input single-output and multivariable control systems using controller sensitivity measures. In R.C.H. Cheng and R.J. Pooley, editors, UKSS'95 - Second Conference of the UK Simulation Society, pages 73-79, Edinburgh, U.K., 1995. UKSS.

[16] P. Eykhoff. System Identification. Wiley, 1974.

[17] D.A. Calahan. Computer-aided Network Design. McGraw-Hill, New York, 1972.

[18] D. Wilkie and W. Perkins. Essential parameters in sensitivity analysis. In Proc. 2nd IFAC symposium on sensitivity and adaptivity, Belgrade, 1968.

[19] Dean Karnopp. Power and energy in linearised systems. Journal of the Franklin Institute, 303(1):85-98, January 1977.

[20] Neil Munro, editor. Symbolic methods in control systems analysis and design. Number 56 in Control engineering series. IEE, Stevenage, UK, 1999.

[21] C. H. An, C. G. Atkeson, and J. M. Hollerbach. Model-based Control of Robot Manipulators. The MIT Press, 1988.

[22] C. Canudas de Wit. Adaptive control for partially known systems. Elsevier, Amsterdam, 1988.

[23] S. Dasgupta, B. D. O. Anderson, and R. J. Kaye. Output error identification methods for partially known systems. Int. J. Control, 43(1):177-191, 1986.

[24] P. J. Gawthrop, R. W. Jones, and S. A. MacKenzie. Identification of partiallyknown systems. Automatica, 28(4):831-836, 1992.

[25] P. J. Gawthrop, J. Ježek, R. W. Jones, and I. Sroka. Grey-box model identification. Control-Theory and Advanced Technology, 9(1), 1993. 
[26] W.H. Press, S.A. Teukolsky, W.T. Vetterling, and B.P. Flannery. Numerical Recipes in C. Cambridge University Press, 2nd edition, 1992.

[27] Peter J. Gawthrop. Estimating physical parameters of nonlinear systems using bond graph models. In Proceedings of the 12th IFAC Symposium on System Identification (SYSID 2000), Santa Barbara, California, USA, June 2000. Available at URL http://www.eng.gla.ac.uk/Control as report number CSC-99003.

[28] MTT. MTT: Model transformation tools. Online WWW Home Page, 2000. URL: http://www.eng.gla.ac.uk/〜peterg/software/MTT/.

[29] G. Rayna. Reduce: Software for Algebraic Computation. Springer, 1987.

[30] Peter J. Gawthrop and Eric Ronco. A sensitivity bond graph approach to estimation and control of mechatronic systems. Control Engineering Practice, to appear, 2000.

[31] F. E. Cellier and J. J. Granda, editors. Proceedings of the 1995 International Conference On Bond Graph Modeling and Simulation (ICBGM'95), volume 27 of Simulation Series, Las Vegas, U.S.A., January 1995. Society for Computer Simulation. 


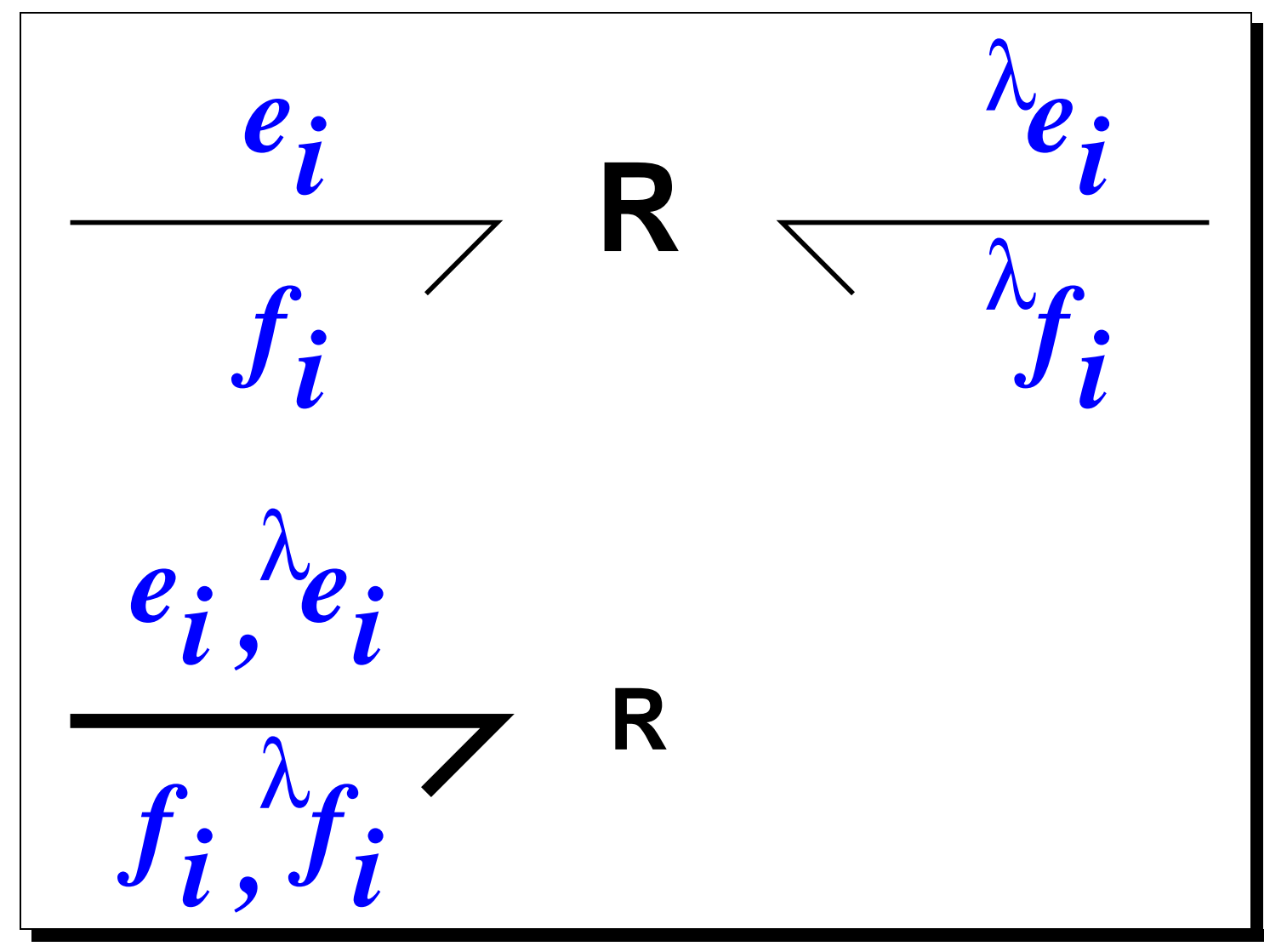

Fig. 1. The sensitivity $\mathbf{R}$ component $\mathbf{s R}$ 


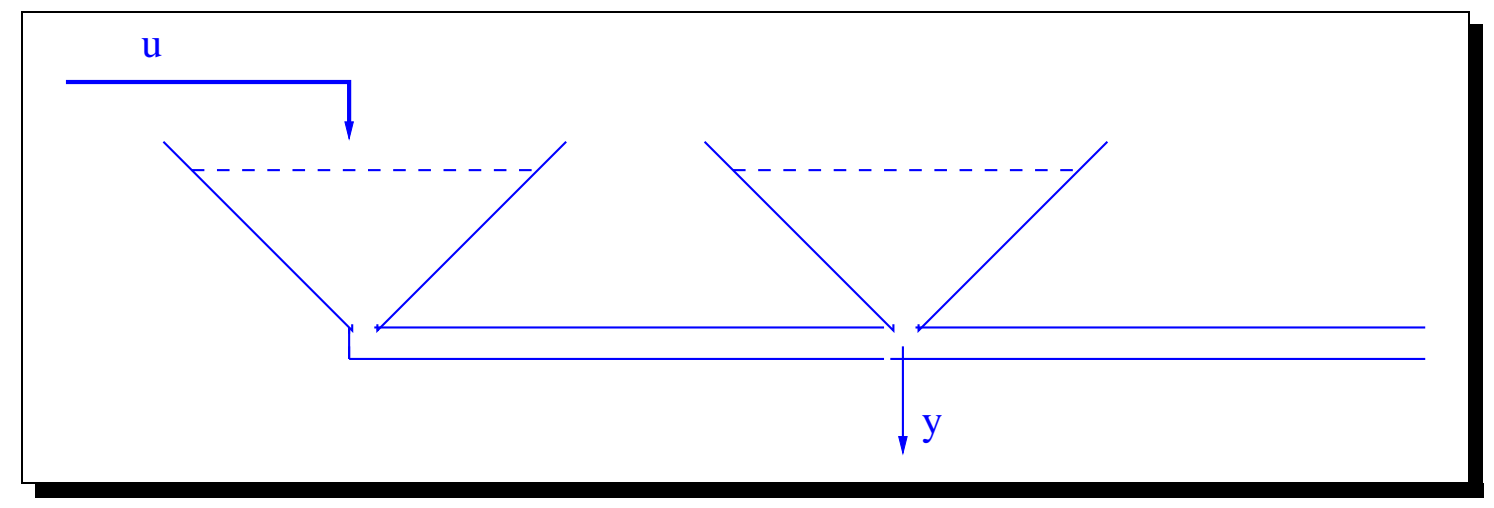

Fig. 2. Two tank system: Schematic 


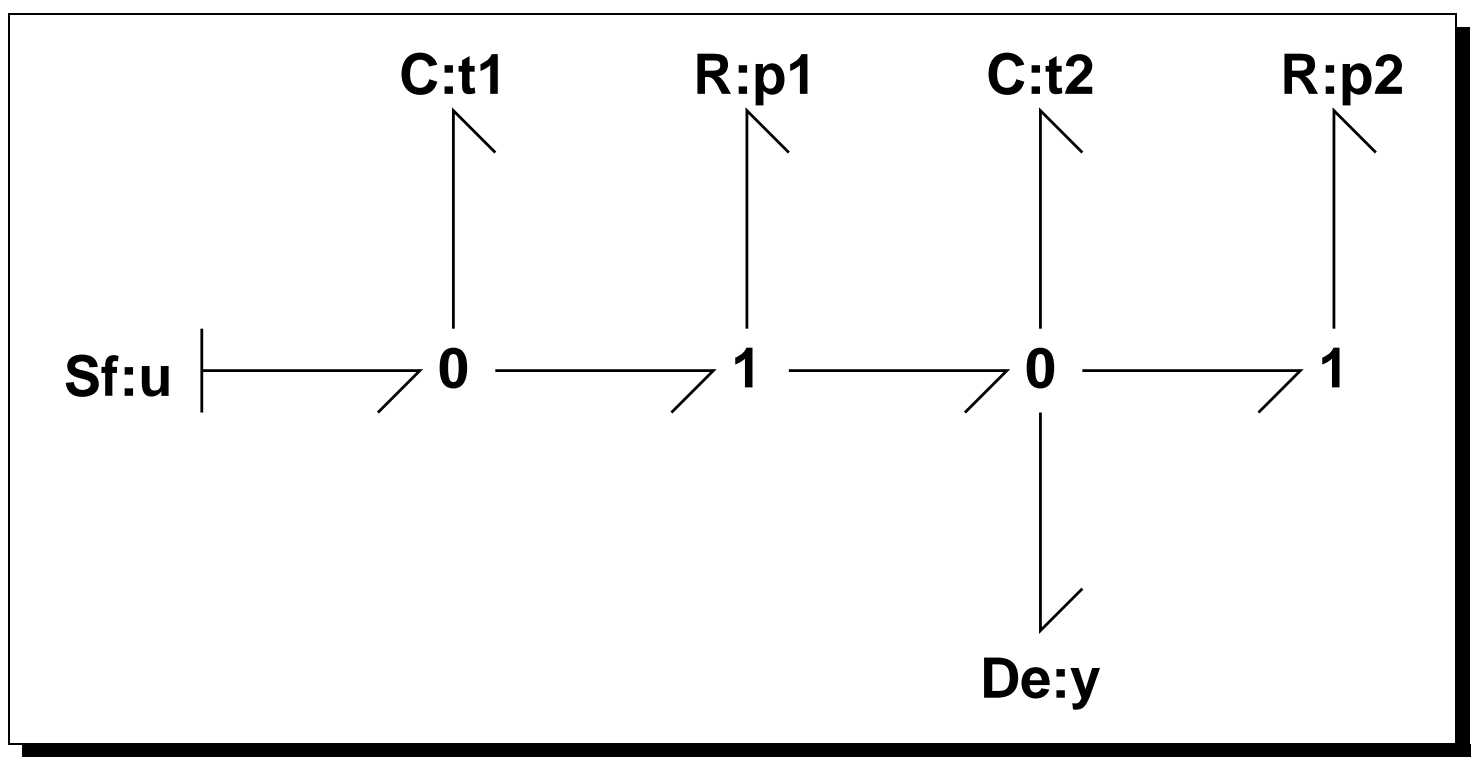

Fig. 3. Two tank system: Bond Graph 


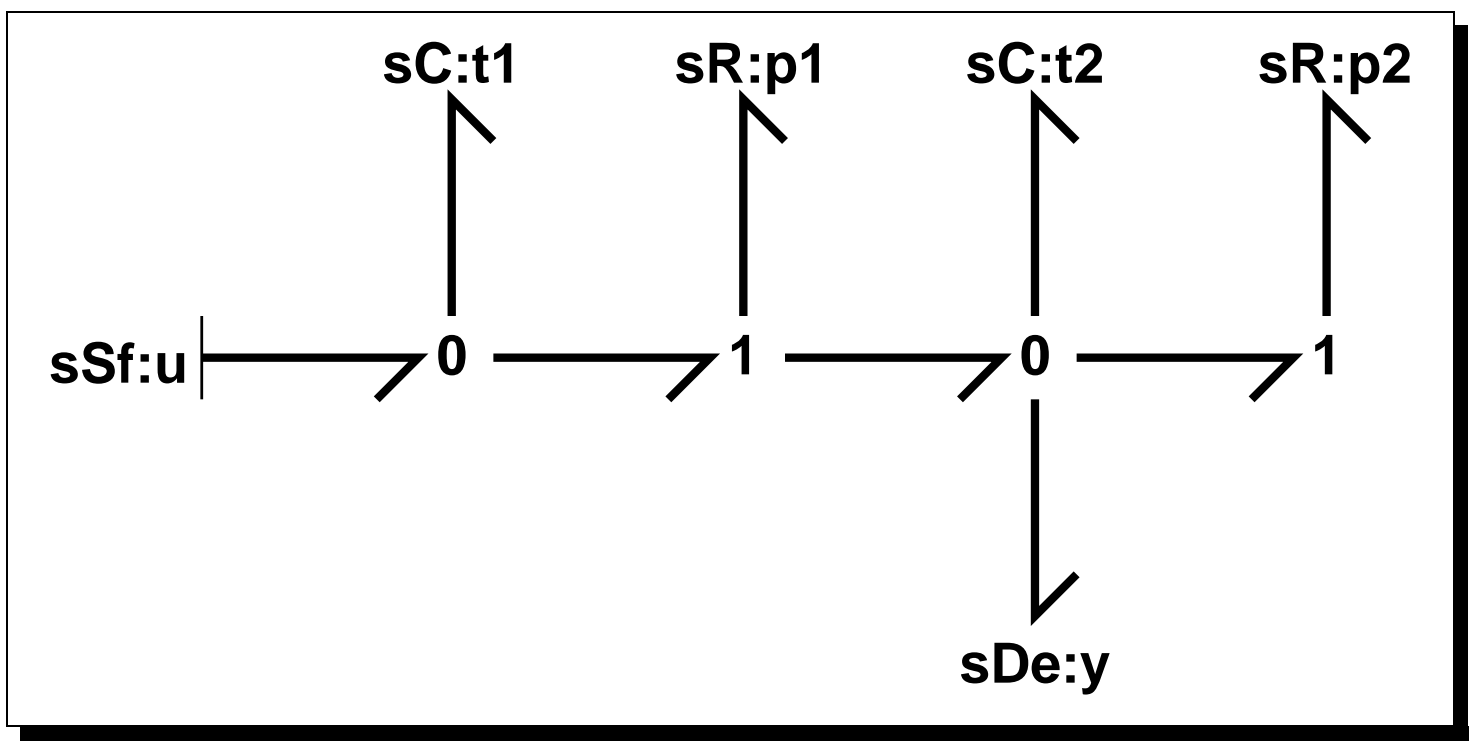

Fig. 4. Two tank system: Sensitivity Bond Graph 


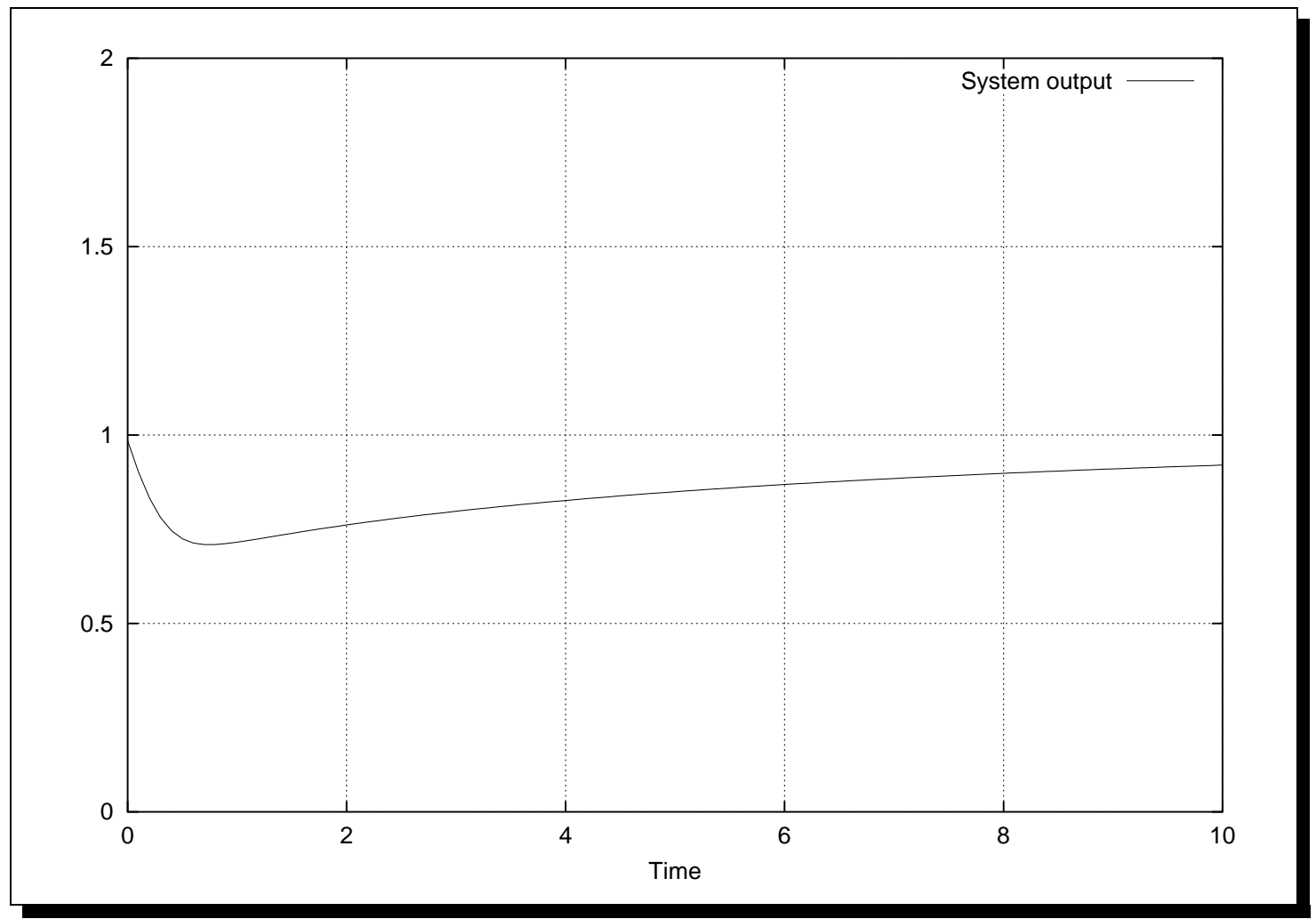

Fig. 5. System output 


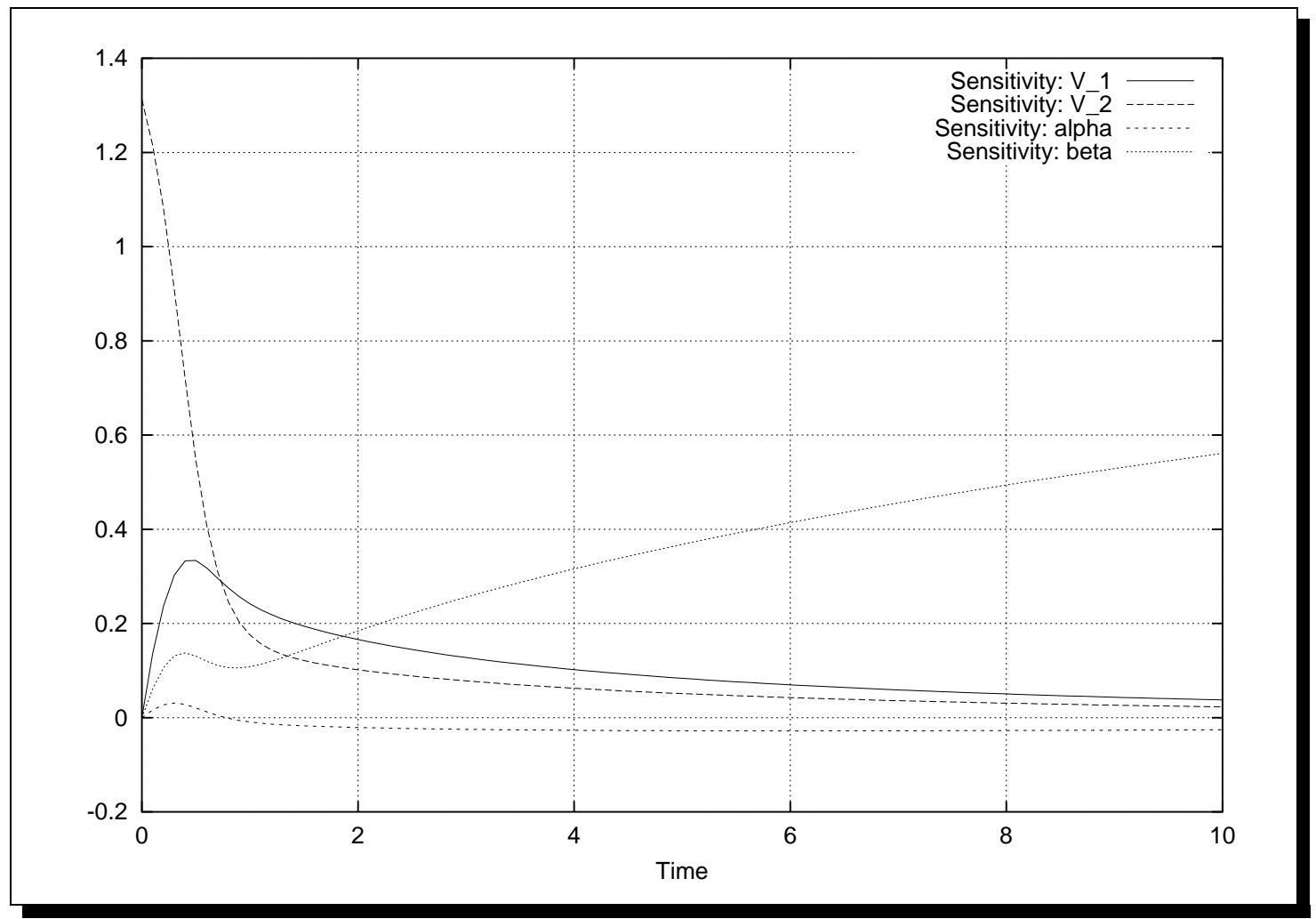

Fig. 6. Model sensitivity functions 


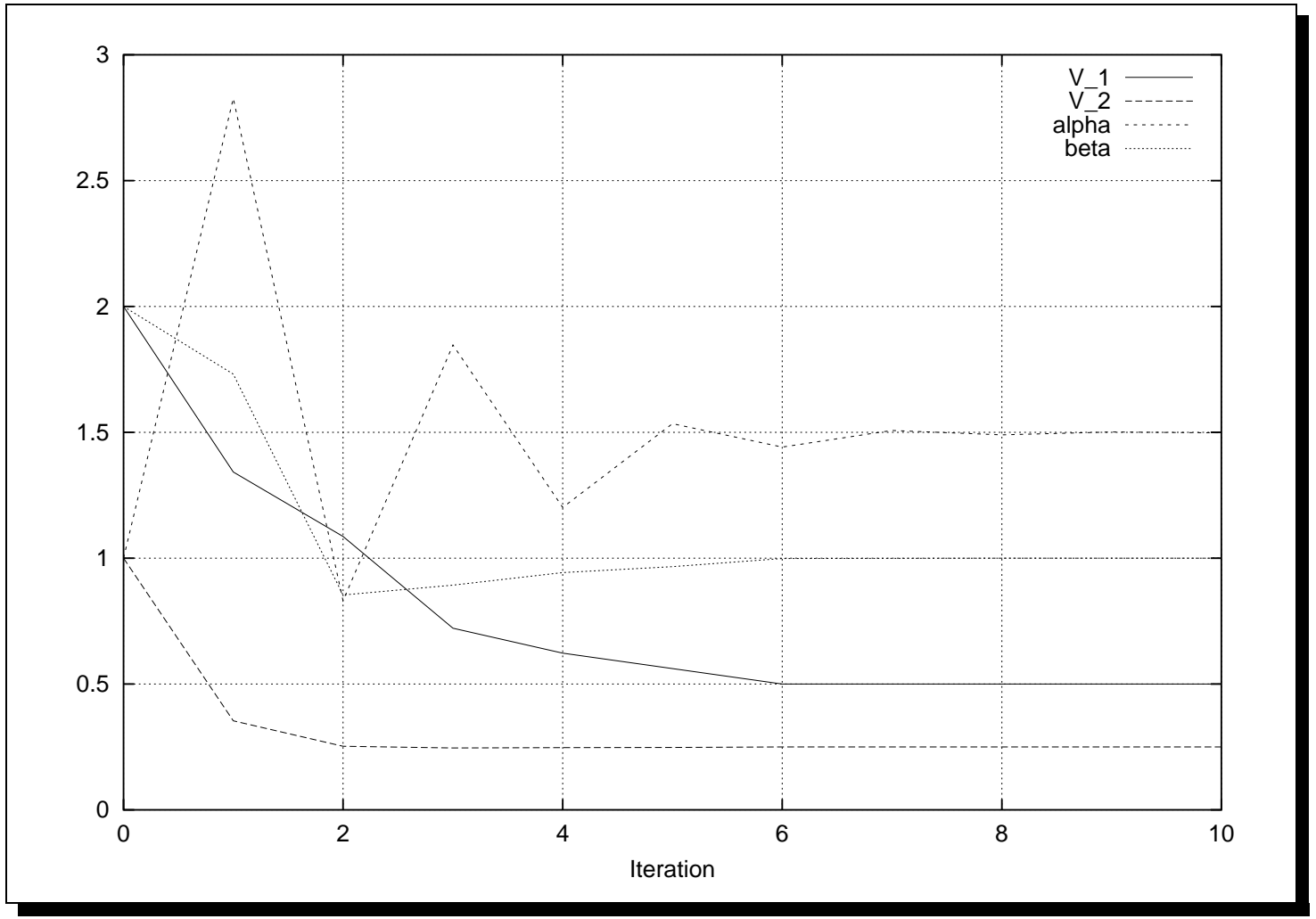

Fig. 7. Estimated parameters 


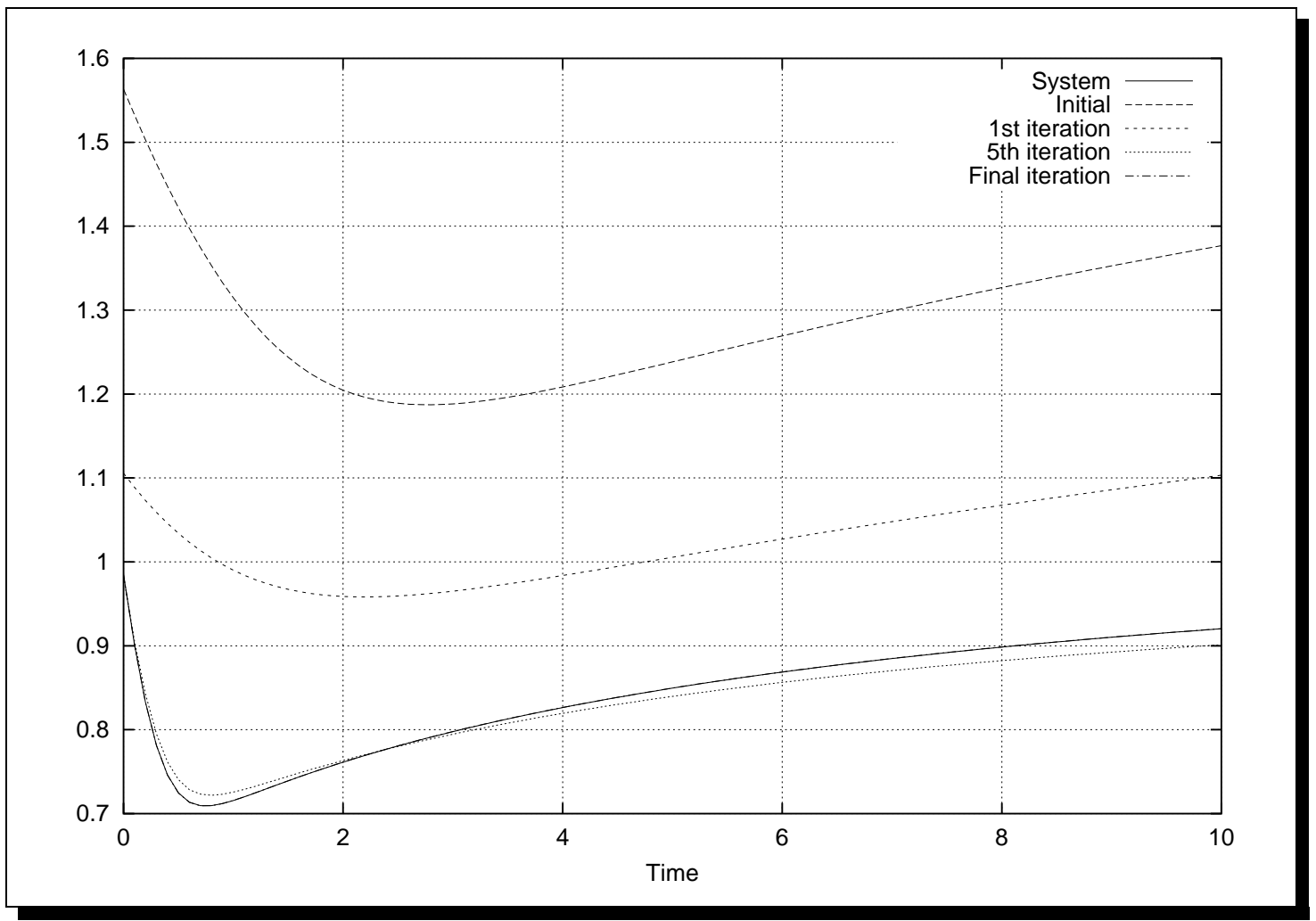

Fig. 8. Estimated output at iterations $0,1,5$ and 10 